nothing but a clouding of the capsule and when I made a division of it she was restored to good sight. That prompted me to make secondary operations in as many cases as I could get hold of. After this capsulotomy there is one objectionable feature that I would like to eliminate, that is the occurrence of glaucoma. It is sometimes acute and comes on within a few days after the operation. Then it is almost always cured. It occurs in about 1 per cent. of all the cases, which is a great deal. In a few cases it is cured by eserin. Glaucoma may occur in such cases any time after the operation. I saw it once in a case of Dr. Agnew after thirty years.

\section{A REPORT OF THIRTY CASES OF CATARACT EXTRACTION WITH REFERENCE TO THE TREATMENT OF PROLAPSE OF IRIS FOLLOWING SIM- PLE EXTRACTION.}

Presented to the Section on Ophthalmology at the Forty-eighth Annual Meeting of the American Medical Association at Philadelphia, Pa., June 1-4, 1897.

BY LOUIS F. LOVE, M.D.

OPHTHALMIC SURGEON TO ST. MARY'S hOSPITAL, ETC. PHILADELPHIA.

Undoubtedly the ideal method of cataract extraction is that in which the lens is removed without iridectomy. But in view of the much better results obtainable by the average operator with iridectomy I am inclined to think that simple extraction should be reserved for selected cases. Certainly it is safe to say that young operators should always use the combined method and wait until their judgment and skill are considerable before attempting simple extraction. The adoption of strict asepsis and antisepsis by the modern ophthalmic surgeon has, no doubt, made simple extraction of cataract more popular at the present day; but the great drawback still remains in the liability of prolapse of the iris coming on after the operation. On my return from Europe two years ago I determined to adopt simple extraction. Twenty out of thirty cataract cases were consecutively operated upon without iridectomy, and in four of them prolapse of the iris ensued in one or more days. This was a large percentage, but even the most skillful surgeons, acknowledge 6 to 9 per cent. It is evident then, that the most careful operator must often face the problem of iris prolapse and the important question arises how best to deal with this very likely complication.

Desiring to ascertain the procedures employed by various prominent surgeons in this country and in Europe, I wrote to a number of them. Among the answers were letters from Dr. de Schweinitz and the late Dr. Keyser of this city, Dr. Knapp and Dr. Noyes of New York, Dr. Fuchs of Vienna and Dr. Trosseau and Dr. de Wecker of Paris, some of whom I will quote, and afterward I will describe my own method somewhat in detail.

Dr. Knapp kindly wrote as follows: "For the last several years I have, or rather Dr. R. O'Born as my associate at the New York Ophthalmic and Aural Institute, has been in the habit of inspecting each eye within twenty-four hours after an extraction, be there symptoms of reaction or not. If there is iris prolapse I cut it at once, reduce the edge of the coloboma and obtain, in almost every case, as undisturbed a recovery as if I had made an iridectomy before or during the extraction. Formerly I inspected eyes the first time, three days after extraction if the patient felt comfortable, and abscised prolapse, if at all, rarely before the tenth day. This gave rise to high degrees of astigmatism on the one hand and to an impure artificial pupil on the other. I mean irregular incarceration of the edges of the coloboma. During the last years I have made simple extraction in relatively more cases than ever before. Prolapse of iris has been very rare, and when it occurred the immediate abscission converted the case into one of combined extraction. I make the abscission in manageable patients under cocain and in nervous and unruly patients under ether."

Dr. Noyes is in the habit of doing an iridectomy as early as possible unless the prolapse is very small.

Dr. de Schweinitz usually follows the rules laid down by Dr. Knapp, whom he quotes: "If the prolapse is discovered within a few hours after its occurrence it is cut off and the edges of the iris reduced exactly as after the operation of iridectomy. If the prolapse is not noted until the third or fourth day it is allowed to remain. Small prolapses may disappear, others produce no irritation, while still others become larger, constricted at their bases or cystoid. These are allowed to remain until the irritation has disappeared and then they are amputated in the same manner as a small staphyloma is abscised." "In a few cases in which there has been some gaping of the wound after the amputation I have inserted a delicate silk suture."

The following is a translation of Dr. Fuchs' letter: "If in the days following the extraction prolapse of the iris occurs I not only cut the iris off, but I cut it out; that is, I draw it a little forward, carry it away with the scissors and go then with the Daviel spoon in both corners of the wound in order to place back the coloboma. I consider the thing ended only when both of the sphincter columns stand equally deep and a regular coloboma exists as if an iridectomy had been done. It is true that this does not succeed perfectly in all cases, as for example, when the patient squeezes the eyelids too much and thereby the vitreous threatens to come out. In cases where subsequent excision of the iris has been done I have seen only one case of iridocyclitis. Otherwise the cases have gone along very nicely. I commenced the simple extraction only two years ago and I still operate on more than half the cases with iridectomy. For this reason I have up to this date only 725 simple extractions to show with about 6 per cent. with prolapse. This would therefore give about forty cases of subsequent excision. Those cases where the iris only late (i.e., after the first eight days) and by degrees pressed itself to the wound, and which in these cases is usually very small, I generally leave untouched."

Drs. Oliver and Risley cut off the prolapse if it is discovered within forty-eight hours, otherwise it is allowed to remain.

In my own cases I have always cut off the prolapse as soon as it is discovered, which fortunately has always been within forty-eight hours. I have also been extremely careful to thoroughly cocainize the eye and to use strict asepsis in every detail, and antisepsis as well, where indicated. In removing the prolapse I used no speculum, but had my assistant employ a retractor and, if needed, the fixation forceps, to pull down the globe. This is somewhat unhandy for the operator, but much safer for the eye. I think that prolapse of the iris could often be avoided if greater care were exercised in the expulsion of cortical matter, clear cortical masses being especially dangerous. 
The preliminary preparation of cataract extraction $/$ cable. Cocain ( 2 per cent.) was always instilled into consisted of having them under control for about two the eye about twenty minutes before operating (and or three days before operation. The whole body, repeated), as this seemed to produce anesthesia of the including the scalp, was bathed in warm water and iris, preventing the patient from squeezing the lids soap bath, the nasal passages carefully washed out during the delivery of lens or during iridectomy. At two or three times daily with an antiseptic wash and the time of the operation the patient's face was thorthe conjunctival sac flooded three times a day with a oughly washed with warm water and soap, followed by saturated solution of boric acid. A purge was given the day before operation.

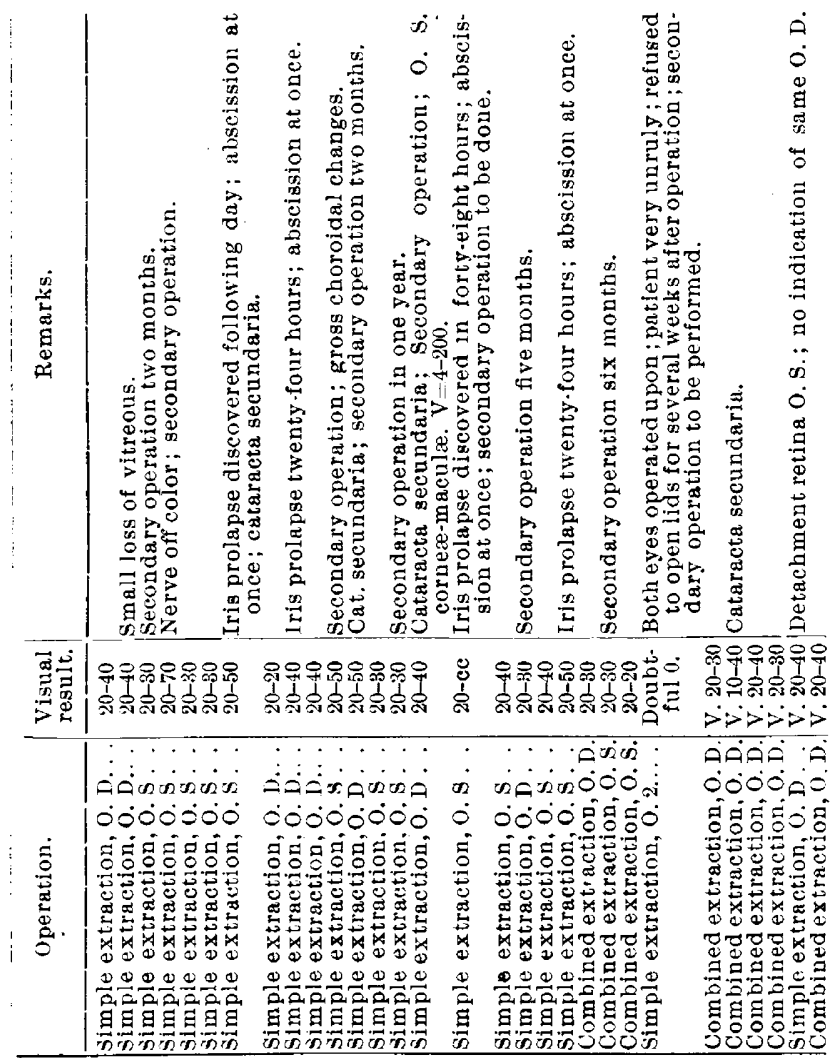

1 to 5,000 solution, and just 2,000 solution was dropped over the globe, which I
believe is the custom of Dr. Knapp. My hands and those of the assistants were carefully looked to. Lately I have used sterilized gloves as employed by Landolt. These are put on immediately after washing the hands in the bichlorid solution and are not removed until the time of the operation.

A very light aseptic gauze bandage with pads (dry) was used in every case. The eye was washed daily with boric acid solution and atropin was instilled. In some cases the bandages were removed in five or six days, and in others not until the fourteenth or fifteenth day. No case was confined to bed more than seven days, four days being about the average.

I am indebted to my assistant, Dr. Martin, and also to the resident physicians of St. Mary's Hospital for the very thorough manner in which they carried out antiseptic detail, as in none of the cases was there the slightest evidence of suppuration.

\section{GLAUCOMA.}

Presented to the Section on Ophthalmology, at the Forty-eighth Anuual Meeting of the American Medical Association, held at Philadelphia, Pa., June 1.4, 1897.

BY DUDLEY S. REYNOLDS, A.M., M.D.

Professor of Ophtbalmology, Otology and Medical Jurisprudence, in the Hospital College of Medicine, Medical Department of the Central University of Kentucky; Surgeon to the Eye and Ear Department of the Louisville City Hospital, and the Gray Street Infirmary, etc.

$$
\text { LOUISVILLE, KY. }
$$

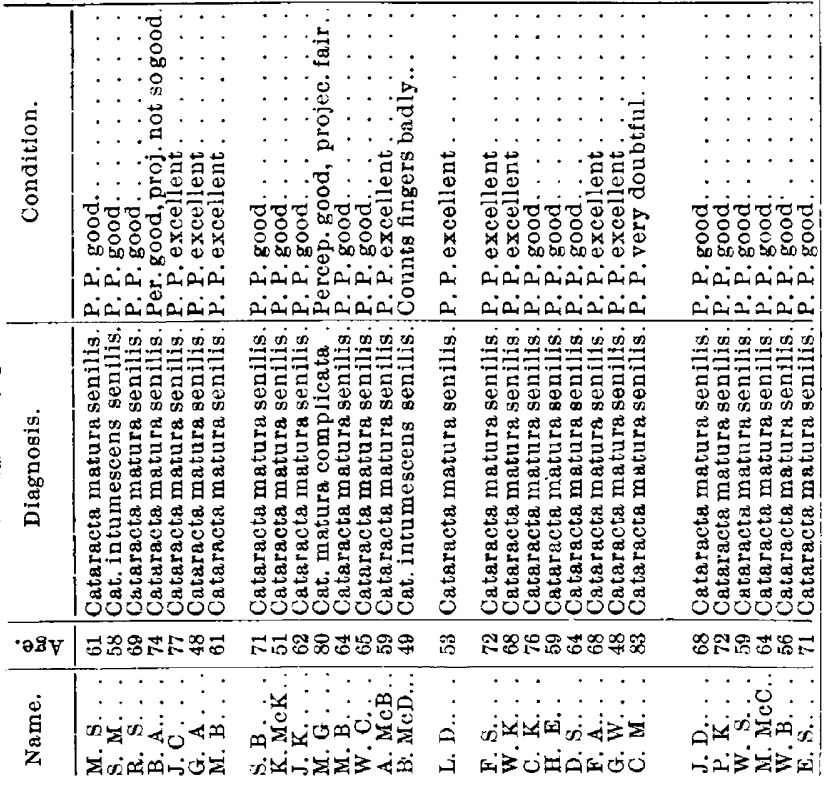

The term glaucoma, which formerly meant seagreen pupillary reflex; with more or less complete blindness, described by Walther as a condition in which the patient sees nothing and the surgeon likewise nothing, has coine to mean so much that it now really means nothing definite. The nearest approach I could come to a location of the structural lesions which give rise to the symptoms of glaucoma would be the ciliary region of the eye. As a matter of fact, disturbances in the nutrition of the ciliary muscle, with or without corresponding disturbances in the ciliary body, presenting all the varying characteristics from a reflex hyperemia to a fulminating parenchymatous inflammation, give rise to some degree of ocular tension, accompanied by undue fulness of the retinal veins and some degree of depression of the surface of the optic disc. Perimetric registration may be required to complete the diagnosis in some mild cases. In acute inflammatory cases this means of diagnosis, if desired, could not be practiced.

The degree of ache varies from the slightest sense of continued discomfort to a sense of excruciating

All instruments except the Graefe knife were sterilized in soda solution. The Graefe knife was dipped in a boiling soda solution and, along with the other instruments, put in absolute alcohol and all carefully wiped with sterilized gauze. The solutions of cocain and boric acid were rendered sterile as far as practipain. Clinically considered, the inflammatory attacks sion, with turbidity of the refracting media, sluggish or entirely absent pupillary movement, with welldefined corneoscleral injection, may safely be accounted manifestations of rheumatism or gout; except- 